\title{
Modulation of Anisotropy on Phonon Transportation in MgS: Theoretical Studies
}

\author{
Li ping $\mathrm{Yao}^{1}$, Hong zhi $\mathrm{Fu}^{1} \&$ Juan $\mathrm{Fu}^{2}$ \\ ${ }^{1}$ College of Physics and Electronic Information, Luoyang Normal College, Luoyang 471022, P. R. China \\ ${ }^{2}$ Zhengzhou municipal design institute, Zhengzhou 450018, People's Republic of China \\ Correspondence: Hong zhi Fu, College of Physics and Electronic Information, Luoyang Normal College, Luoyang \\ 471022, P. R. China. Tel: 86-0379-6551-5016. E-mail: fhzscdx@163.com
}

\author{
Received: November 19, 2014 Accepted: December 15, $2014 \quad$ Online Published: December 27, 2014 \\ doi:10.5539/jmsr.v4n2p1 URL: http://dx.doi.org/10.5539/jmsr.v4n2p1
}

\begin{abstract}
The anisotropy and phonon focusing of $\mathrm{MgS}$ are investigated through continuously elastic medium theory and physical acoustics theory in the solid state. The anisotropy and phonon propagation are theoretically researched in three-dimensional figures and their projections. The contours of three dimensional slowness surfaces give insights into the mixing of longitudinal modes and transverse modes, and show the origin of the phonon caustics due to the fact that the phonon phase velocity and group velocity are generally not collinear in elastically anisotropic crystals. The comparative investigations between anisotropy and slowness show that the propagation of elastic wave is affected by the elastic anisotropy of the lattice, and the phonon caustics "walk along" the extreme direction of elastic anisotropy.
\end{abstract}

Keywords: theoretical studies, elastic properties, acoustical properties of solids

\section{Introduction}

Belonging to the wide-gap binary $\mathrm{A}^{\mathrm{N}} \mathrm{B}^{8-\mathrm{N}}$ semiconductors, the properties of II-VI compounds have attracted the interest of researchers for over 30 years because of their wide band gap character and the potential applications for optoelectronic devices (Côté, Zakharov, Rubio, \& Cohen, 1997; Lee \& Chang, 1995). Because of the technological interests, the feasibility of green-blue opto-electronic devices based on these materials has been demonstrated (Drief, Tadjer, Mesri, \& Aourag, 2004).

$\mathrm{MgS}$ forms a very important closed-shell ionic system crystallized in the NaCl-type (B1) structure at ambient conditions (Chakrabarti, 2000; Varshney, Kaurav, Sharma, \& Singh, 2008) and crystallize in four fold-coordinated cubic zinc-blende structure (B3) at room temperature (Zimmer, Winzen, \& Syassen, 1985). MgS is a wide-band-gap semiconductor (the band gap certainly exceeds $4.5 \mathrm{eV}$ at room temperature). Unlike $\mathrm{ZnSe}$ and $\mathrm{ZnTe}$ which have been extensively studied, $\mathrm{MgS}$ is one of the least studied members of this family (Chen, Xiao, \& Zu, 2005; Duman, Bagc, Tutuncu, \& Srivastava, 2006; Froyen, Wei, \& Zunger, 1988; Lichanot, Dargelos, Larrie, \& Orlando, 1994).

The phase transition of MgS was studied (Kalpana, Palanivel, Thomas, \& Rajagopalan, 1996; Sahraoui, Zerroug, Louail, \& Maouche, 2007) based on linear muffin-tin orbital method in its tight binding representation (TBLMTO) and the generalized gradient approximation within the density functional theory (DFT). The magnesium of MgS has been investigated (Rached et al., 2003) using the full-potential linear muffin-tin orbital (FP-LMTO) method augmented by a plane wave (PLW) basis. In theory, the electronic structures (Ching, Gan, \& Huang, 1995) and lattice dynamics (Wolverson et al., 2001) of MgS have been studied using the full potential linearised augmented plane wave (FPLAPW) method and tight binding linear muffin tin orbital method, respectively.

Despite much theoretical works, MgS is so far not well understood for some of its properties, for example, the effect of elastic anisotropy on the acoustic energy propagation (Taylor, Maris, \& Elbaum, 1969; Hensel \& Dynes, 1977; Maris \& Acoust, 1971; Maris, 1983). This has motivated us to examine the nature of acoustic-wave propagation in elastically anisotropic solids, with emphasis on the phonon focus (Northrop \& Wolfe, 1980; Wolfe, 1998; Hurley, Wolfe, \& McCarthy, 1986; Hurley \& Wolfe, 1985). Still today, these properties remain as a source for investigation and for possible new discoveries (Weilert, Msall, Anderson, \& Wolfe, 1993; Every, Sachse, Kim, \& Thompson, 1990; Hauser, Weaver, \& Wolfe, 1992; Würz, Wesner, Hillmann, \& Grill, 1995). In the present paper, the physical and elastic anisotropy of $\mathrm{MgS}$ will be discussed, and the wave propagation will be described for the slowness surface and group velocity. 


\section{Methodology}

The stiffness constants $C_{\mathrm{ij}}$ are performed based on the plane-wave pseudopotential density function theory (DFT) (Hohenberg \& Kohn, 196; Kohn \& Sham, 1965). Vanderbilt-type ultrasoft pseudopotentials (USPP) (Vanderbilt, 1990) are employed to describe the electron-ion interactions. The effects of exchange correlation interaction are treated with the generalized gradient approximation (GGA) of Perdew-Burke-Eruzerhof(PBE) (Perdew, Burke, \& Ernzerhof, 1996). In the structure calculation, a plane-wave basis set with energy cut-off $460.00 \mathrm{eV}$ is used. Pseudo-atomic calculations are performed for $\mathrm{Mg} 3 \mathrm{~s}^{2}$ and $\mathrm{S} 3 \mathrm{~s}^{2} 3 \mathrm{p}^{6}$. For the Brillouin-zone sampling, we adopt the $6 \times 6 \times 6$ Monkhorst-Pack k-point grid (Monkhorst, \& Pack, 1976) with which the total energies are converged to $2.0 \times 10^{-5} \mathrm{eVper}$ atom and the forces to $5.0 \times 10^{-2} \mathrm{eV} / \AA$.

The elastic compliance $S_{\mathrm{ij}}$ can be calculated by the stiffness constants $C_{\mathrm{ij}}$ for cubic symmetry classes:

$$
\begin{aligned}
& S_{11}=\frac{C_{11}+C_{12}}{\left(C_{11}-C_{12}\right)\left(C_{11}+2 C_{12}\right)} ; \\
& S_{12}=\frac{-C_{12}}{\left(C_{11}-C_{12}\right)\left(C_{11}+2 C_{12}\right)} ; \\
& S_{44}=\frac{1}{C_{44}} ;
\end{aligned}
$$

In order to obtain the orientation dependence of elastic moduli, we need the Bond stress transformation matrices $[M]$ and $[N]$ (Bond, 1976) to get the stiffness and compliance transformation matrices $\left[C^{\prime}\right]=[M] \cdot[C] \cdot[M]^{T}$ and $\left[S^{\prime}\right]=[N] \cdot[C] \cdot[N]^{T}$, respectively.

and

$$
M=\left[\begin{array}{cccccc}
\alpha_{x x}^{2} & \alpha_{x y}^{2} & \alpha_{x z}^{2} & 2 \alpha_{x y} \alpha_{x z} & 2 \alpha_{x z} \alpha_{x x} & 2 \alpha_{x x} \alpha_{x y} \\
\alpha_{y x}^{2} & \alpha_{y y}^{2} & \alpha_{y z}^{2} & 2 \alpha_{y y} \alpha_{y z} & 2 \alpha_{y z} \alpha_{y x} & 2 \alpha_{y x} \alpha_{y y} \\
\alpha_{z x}^{2} & \alpha_{z y}^{2} & \alpha_{z z}^{2} & 2 \alpha_{z y} \alpha_{z z} & 2 \alpha_{z z} \alpha_{z x} & 2 \alpha_{z x} \alpha_{z y} \\
\alpha_{y x} \alpha_{z x} & \alpha_{y y} \alpha_{z y} & \alpha_{y z} \alpha_{z z} & \alpha_{y y} \alpha_{z z}+\alpha_{y z} \alpha_{z y} & \alpha_{y x} \alpha_{z z}+\alpha_{y z} \alpha_{z x} & \alpha_{y y} \alpha_{z x}+\alpha_{y x} \alpha_{z y} \\
\alpha_{z x} \alpha_{x x} & \alpha_{z y} \alpha_{x y} & \alpha_{z z} \alpha_{x z} & \alpha_{x y} \alpha_{z z}+\alpha_{x z} \alpha_{z y} & \alpha_{x z} \alpha_{z x}+\alpha_{x x} \alpha_{z z} & \alpha_{x x} \alpha_{z y}+\alpha_{x y} \alpha_{z x} \\
\alpha_{x x} \alpha_{y x} & \alpha_{x y} \alpha_{y y} & \alpha_{x z} \alpha_{y z} & \alpha_{x y} \alpha_{y z}+\alpha_{x z} \alpha_{y y} & \alpha_{x z} \alpha_{y x}+\alpha_{x x} \alpha_{y z} & \alpha_{x x} \alpha_{y y}+\alpha_{x y} \alpha_{y x}
\end{array}\right]
$$

$$
N=\left[\begin{array}{cccccc}
\alpha_{x x}^{2} & \alpha_{x y}^{2} & \alpha_{x z}^{2} & \alpha_{x y} \alpha_{x z} & \alpha_{x z} \alpha_{x x} & \alpha_{x x} \alpha_{x y} \\
\alpha_{y x}^{2} & \alpha_{y y}^{2} & \alpha_{y z}^{2} & \alpha_{y y} \alpha_{y z} & \alpha_{y z} \alpha_{y x} & \alpha_{y x} \alpha_{y y} \\
\alpha_{z x}^{2} & \alpha_{z y}^{2} & \alpha_{z z}^{2} & \alpha_{z y} \alpha_{z z} & 2 \alpha_{z z} \alpha_{z x} & 2 \alpha_{z x} \alpha_{z y} \\
2 \alpha_{y x} \alpha_{z x} & 2 \alpha_{y y} \alpha_{z y} & 2 \alpha_{y z} \alpha_{z z} & \alpha_{y y} \alpha_{z z}+\alpha_{y z} \alpha_{z y} & \alpha_{y x} \alpha_{z z}+\alpha_{y z} \alpha_{z x} & \alpha_{y y} \alpha_{z x}+\alpha_{y x} \alpha_{z y} \\
2 \alpha_{z x} \alpha_{x x} & 2 \alpha_{z y} \alpha_{x y} & 2 \alpha_{z z} \alpha_{x z} & \alpha_{x y} \alpha_{z z}+\alpha_{x z} \alpha_{z y} & \alpha_{x z} \alpha_{z x}+\alpha_{x x} \alpha_{z z} & \alpha_{x x} \alpha_{z y}+\alpha_{x y} \alpha_{z x} \\
2 \alpha_{x x} \alpha_{y x} & 2 \alpha_{x y} \alpha_{y y} & 2 \alpha_{x z} \alpha_{y z} & \alpha_{x y} \alpha_{y z}+\alpha_{x z} \alpha_{y y} & \alpha_{x z} \alpha_{y x}+\alpha_{x x} \alpha_{y z} & \alpha_{x x} \alpha_{y y}+\alpha_{x y} \alpha_{y x}
\end{array}\right]
$$

Where $\alpha_{i j}$ are the directional cosine at an angle between the $x_{i}$-axis and $x_{p}^{\prime}$-axis, $[M]^{T}$ and $[N]^{T}$ are the transposed matrices of $[M]$ and $[N]$ respectively.

Some elastic properties (shear modulus and Poisson's ratio) require two mutually perpendicular unit vectors. The first unit vector, $l$, is used to represent the uniaxial stress, and is shown advantageously by two angles $(\theta, \varphi)$. Another unit vector, $m$, perpendicular to the first unit vector $l$, is characterized by the angle $\chi(0,2 \pi)$, followed by the Figure 1.

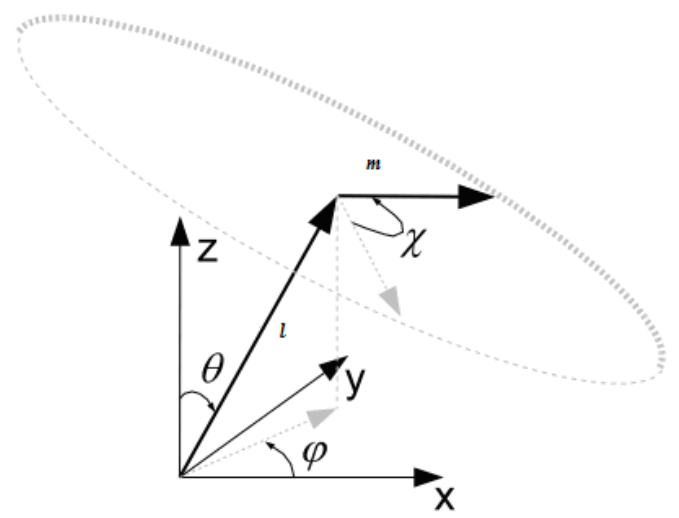

Figure 1. the coordinates of the two vectors are used to calculate to the shear modulus and Poisson's ratio 
The coordinates of the two vectors are,

$$
l=\left(\begin{array}{l}
\sin \theta \cos \varphi \\
\sin \theta \sin \varphi \\
\cos \theta
\end{array}\right) ; \quad m=\left(\begin{array}{l}
\cos \theta \cos \varphi \cos \chi-\sin \varphi \sin \chi \\
\cos \theta \sin \varphi \cos \chi+\cos \varphi \sin \chi \\
-\sin \theta \cos \chi
\end{array}\right)
$$

Using the above theory, the Young's modulus in the $x_{1}^{\prime}$-direction is represented by the reciprocal of $s_{1111}^{\prime}$, i.e.

$$
\frac{1}{Y^{\prime}}=s_{1111}^{\prime}=\sum_{i} \sum_{j} \sum_{k} \sum_{l} \alpha_{1 i} \alpha_{1 j} \alpha_{1 k} \alpha_{1 l} s_{i j k l}
$$

The shear modulus in the $x_{1}^{\prime}$-direction on the $x_{2}^{\prime}$-plane or in the $x_{2}^{\prime}$-direction on the $x_{1}^{\prime}$-plane is given by the reciprocal of $4 s_{1212}^{\prime}$, i.e.

$$
\frac{1}{G^{\prime}}=4 s_{1212}^{\prime}=4 \sum_{i} \sum_{j} \sum_{k} \sum_{l} \alpha_{1 i} \alpha_{2 j} \alpha_{1 k} \alpha_{2 l} s_{i j k l}
$$

When a uniaxial stress is applied in the $x_{1}^{\prime}$-direction, Poisson's ratio in the $x_{2}^{\prime}$-direction is given by:

$$
\sigma_{12}^{\prime}=-\frac{\varepsilon_{22}^{\prime}}{\varepsilon_{11}^{\prime}}=-\frac{s_{1122}^{\prime}}{s_{1111}^{\prime}}=-\frac{\sum_{i} \sum_{j} \sum_{k} \sum_{l} \alpha_{1 i} \alpha_{1 j} \alpha_{2 k} \alpha_{2 l} s_{i j k l}}{\sum_{i} \sum_{j} \sum_{k} \sum_{l} \alpha_{1 i} \alpha_{1 j} \alpha_{1 k} \alpha_{1 l} s_{i j k l}}
$$

Therefore, after the transformation formulas of the coordinates for the compliance-constant matrix $\left\{s_{i j k l}\right\}$, the Young's modulus, the shear modulus, and Poisson's ratio are obtained for a single crystal in different orientations. The wave propagation in elastic continuum theory is governed by the well-known Christoffel equation (Koos \& Wolfe, 1984):

$$
k^{2}\left[\begin{array}{lll}
\tau_{11} & \tau_{12} & \tau_{13} \\
\tau_{21} & \tau_{22} & \tau_{23} \\
\tau_{31} & \tau_{32} & \tau_{33}
\end{array}\right]\left[\begin{array}{c}
v_{1} \\
v_{2} \\
v_{3}
\end{array}\right]=\rho \omega^{2}\left[\begin{array}{c}
v_{1} \\
v_{2} \\
v_{3}
\end{array}\right]
$$

where $v$ is the phase velocity, $\omega$ is constant frequency, $k$ is the wave vector, and $\rho$ is the density. The Christoffel coefficients are given by $\tau_{i j}=C_{i k j m} n_{k} n_{m}$, where $C_{i k j m}$ is a fourth-rank tensor describing the elastic constants, and $n_{k}$ is the direction cosine of the wave vector $\mathbf{k}$.

\section{Discussion}

\subsection{The elastic Properties of $M g S$}

The calculated elastic compliance constants $\left(S_{\mathrm{ij}}\right)$ and elastic constants $\left(C_{\mathrm{ij}}\right)$ of $\mathrm{MgS}$ with $\mathrm{B}_{1}$ phase are listed in Table 1 together with other theoretical results.

Table 1. The calculated elastic constants $C_{\mathrm{ij}}(\mathrm{GPa})$ of $\mathrm{MgS}$.

\begin{tabular}{llccc}
\hline This work & \multicolumn{3}{c}{ Other theoretical calculations } \\
\hline$C_{11}$ & 89 & $183.45^{[\mathrm{a}]}$ & $104.2^{\mathrm{GGA}[\mathrm{b}]}$ & $113.7^{\mathrm{LDA}[\mathrm{b}]}$ \\
$C_{12}$ & 55 & $34.9^{[\mathrm{a}]}$ & $81.3^{\mathrm{GGA}[\mathrm{b}]}$ & $75.5^{\text {LDA [b] }}$ \\
$C_{44}$ & 39 & $69.01^{[\mathrm{a}]}$ & $12.7^{\mathrm{GGA}[\mathrm{b}]}$ & $14.5^{\text {LDA [b] }}$ \\
\hline
\end{tabular}

Source: [a], Drief, Tadjer, Mesri, \& Aourag, 2004; [b], Rached et al., 2003

In Figure 2, the three-dimensional contours are carefully presented for Young's modulus $(Y)$, shear modulus $(G)$ and Poisson's ratio $(\sigma)$ as well as the projections in different planes $((100)$ and $(\overline{1} 10)$ planes). 


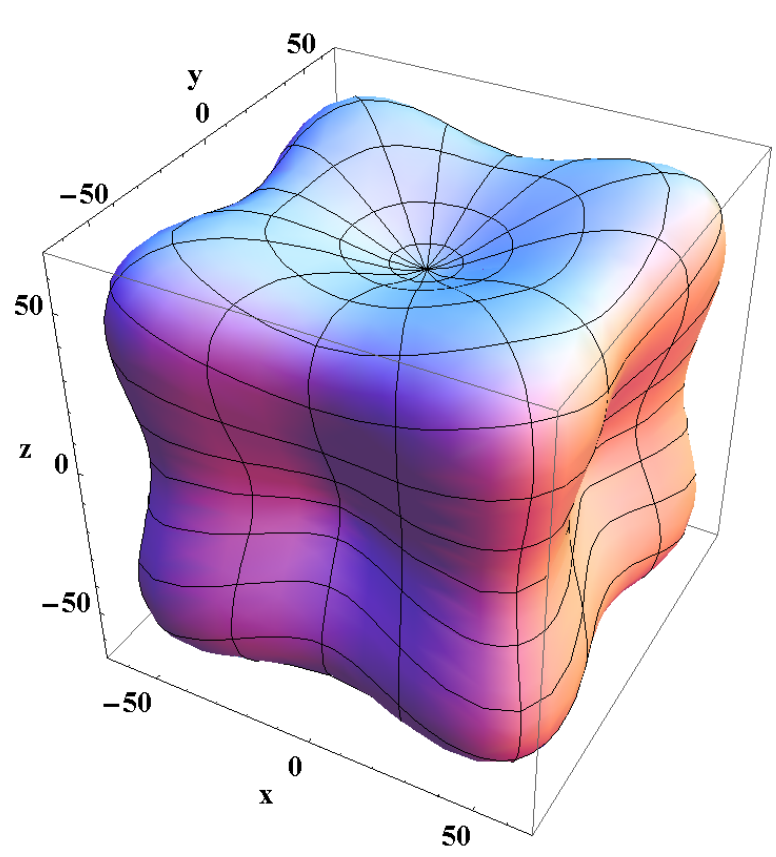

Figure 2a. Orientation dependence of Young's odulus

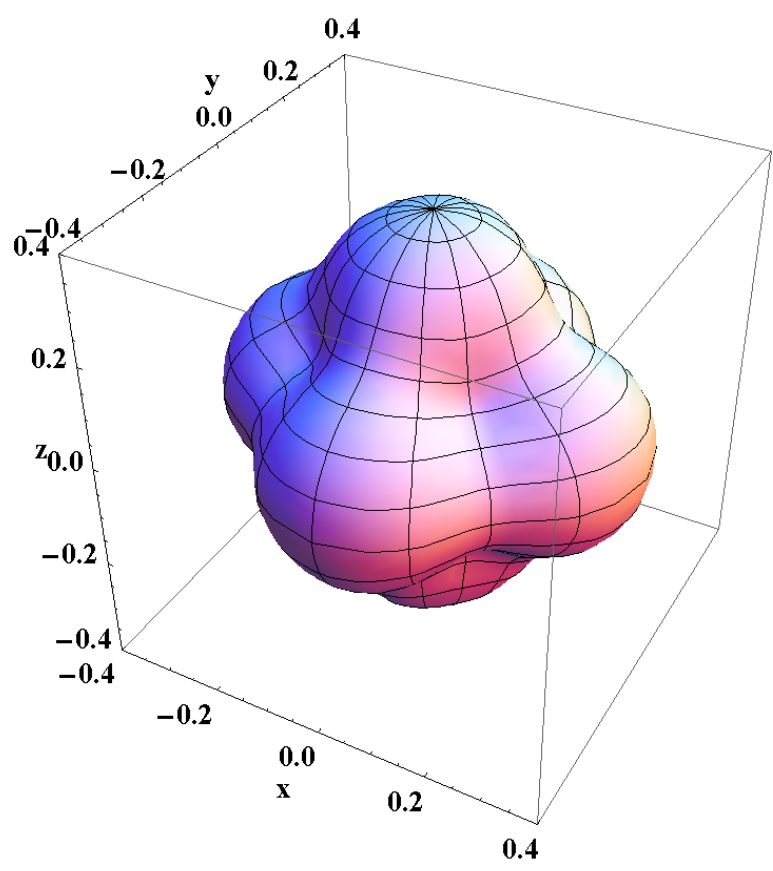

Figure $2 c$. Orientation dependence of Poisson's ratio

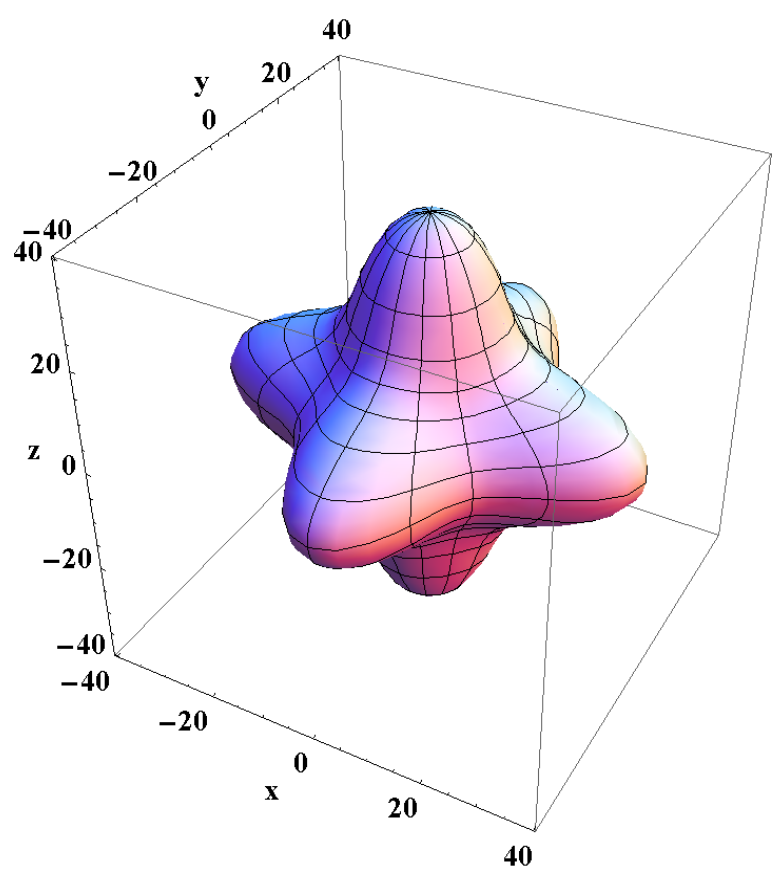

Figure $2 b$. Orientation dependence of shear modulus

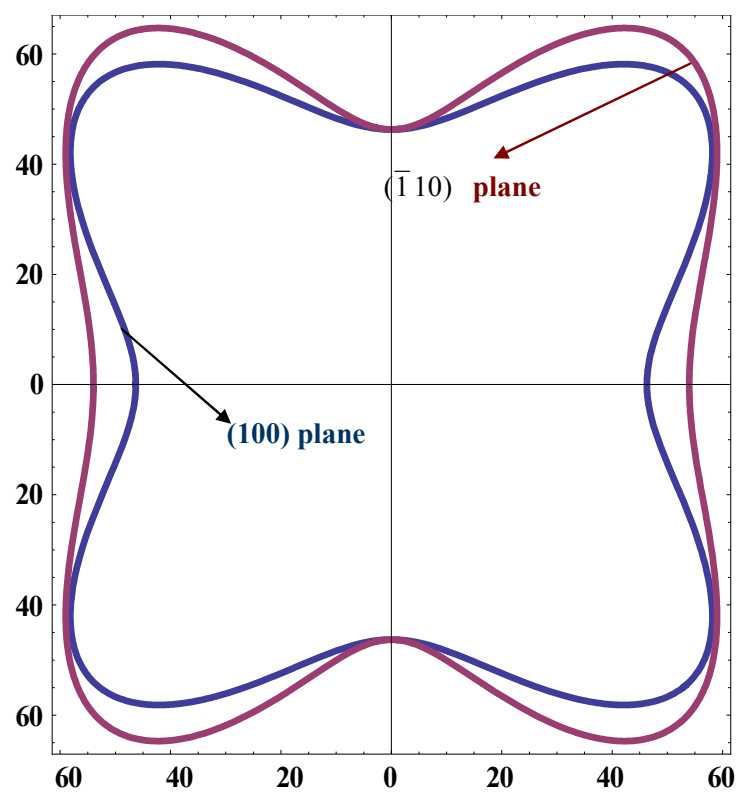

Figure $2 d$. Orientation dependence of Young's modulus in (100) and (110) planes 


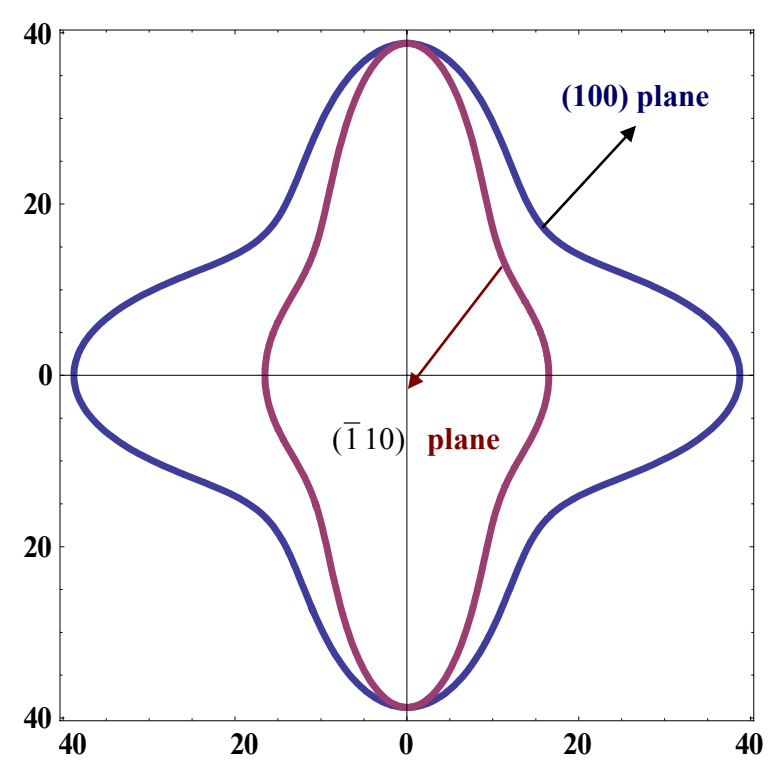

Figure $2 e$. Orientation dependence of shear modulus in (100) and ( $\overline{1} 10)$ planes

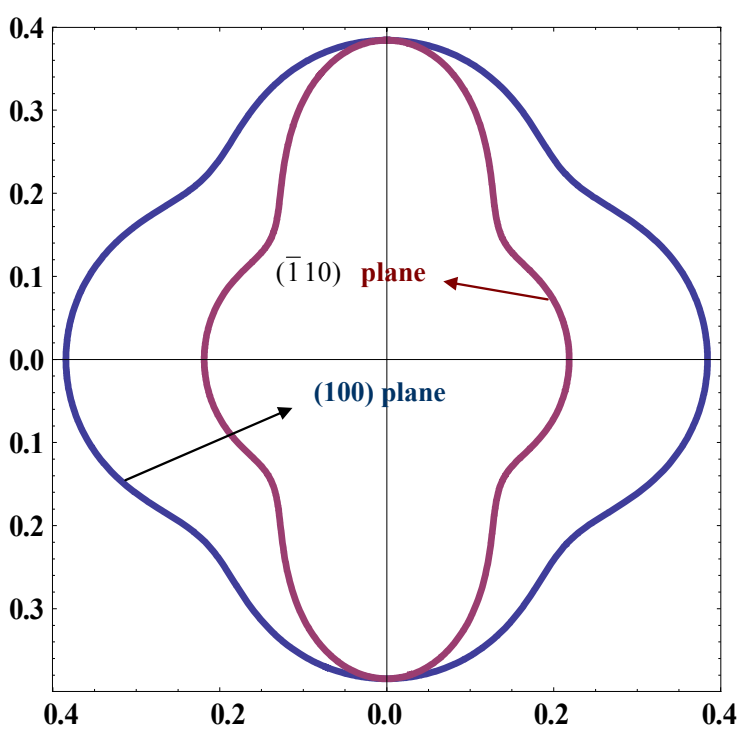

Figure $2 f$. Orientation dependence of Poisson's ratio in (100) and (1 10) planes

Figure 2. (color on line) Orientation dependence of Young's modulus (2a), shear modulus (2b) and Poisson's ratio (2c) for $\mathrm{MgS}$, together with the projections in different planes $(2 d-f)$, the unit is GPa for Young's modulus and shear modulus

The directional dependence of $Y$ depends remarkably upon the crystal orientation. The strain produced in some directions in the crystal will be different in magnitude for the same longitudinal stress applied in one direction due to the fact that anisotropy in the elastic properties of a crystal can be demonstrated by plotting the Young's modulus surface, shear modulus surface and Poisson's ratio surface. Sections of these surfaces together with their projections are shown in Figure 2. Contours of $Y$ surface along the symmetry planes (100) and $\overline{1} 10$ planes are plotted in Figure $2 d$. The Young's modulus minimum in the (100) plane and maximum in the [111] direction can be clearly seen. This indicates that the strain produced along the [100] direction is smaller compared to other directions when applying stress, which indicates the presence of very strong intermolecular forces of attraction in this direction. This behavior is due to the fact that the interatomic distance of $\mathrm{Mg}-\mathrm{S}(2.609 \AA)$ in the [100] direction is shorter than that of $\mathrm{Mg}-\mathrm{S}(4.520 \AA)$ in the [111] direction. This feature also indicates that the directional dependence of $Y$ is strongly affected by the atomic arrangement of the crystal. In Figure $2 b$ and $e$ we also show the orientation dependence of shear modulus. The relationship can be clearly seen, i.e., $G_{[100]}>G_{[011]}>G_{[111]}$, which shows that the shear modulus exhibits a high value in the atomic close-packed direction. The Poisson's ratio provides more information about the characteristics of the bonding forces than any of the other elastic constants, and is not easily calculated for the three-dimensional contour. The $\sigma$ varies over a large range of values as shown in Figure $2 c$ and $f$, which the features of $\sigma$ are $0.304<\sigma<0.383$ in (100) plane and $0.198<\sigma<0.383$ in $\overline{1} 10$ plane. It means that when the stress is applied in the direction perpendicular to the (100) and $\overline{1} 10$ plane, the maximum strain is observed in the [100] direction and the minimum strain in the [111] direction. There are two anisotropy for a cubic lattice structure, i.e., Zener (1948) and Every (1980) anisotropy followed by $A_{\mathrm{z}}=2 C_{44} /\left(C_{11}-C_{12}\right)$ and $A_{\mathrm{E}}=$ $\left(C_{11}-C_{12}-2 C_{44}\right) /\left(C_{11}-C_{44}\right)$. They give the anisotropic variation of the sound velocity between the displacement vector $\mathbf{u}$ and the wave vector $\mathrm{k}$. Because $A_{\mathrm{z}}$ and $A_{\mathrm{E}}$ are not trivially related, i.e., $A_{\mathrm{z}}$ can not be expressed in terms of $A_{\mathrm{E}}$. Thus, there is no unique measure of elastic anisotropy even in the simple case of cubic lattice symmetry. For isotropic systems, $A_{\mathrm{z}}=1$, and $A_{\mathrm{E}}=0$.

The pure transverse and longitudinal slowness can be calculated with $A_{\mathrm{z}}$ [37]:

$$
\left(\frac{k}{\omega}\right)_{S T}=\sqrt{\frac{2 \rho A_{z}}{2 c_{44}}},\left(\frac{k}{\omega}\right)_{F T}=\sqrt{\frac{2 \rho}{A_{z}\left(c_{11}-c_{12}\right)}},\left(\frac{k}{\omega}\right)_{L}=\sqrt{\frac{\rho}{\left(1-\frac{1}{A_{z}}\right)\left(c_{11}+c_{44}\right)}}
$$


There are two interesting situations to appear, i.e., when $A_{\mathrm{z}}>1$, the quasi-shear slowness surface appears outward convex, and the quasi-longitudinal slowness surface appears inside concave; the case is just opposite when $A_{\mathrm{z}}<1$. The calculated $A_{\mathrm{z}}$ of $\mathrm{MgS}$ is 2.29 . The 3D $A_{\mathrm{z}}$ figure together with its projection is shown in Figure 3. The huge anisotropy can be seen in the [100], [111] and [011] direction (Figure 3a). In (100) and ( 10 ) planes, the anisotropy appears different, i.e., the former is higher than the latter (Figure $3 b$ ). This is attributed to the different distribution of atoms in the different directions and planes.

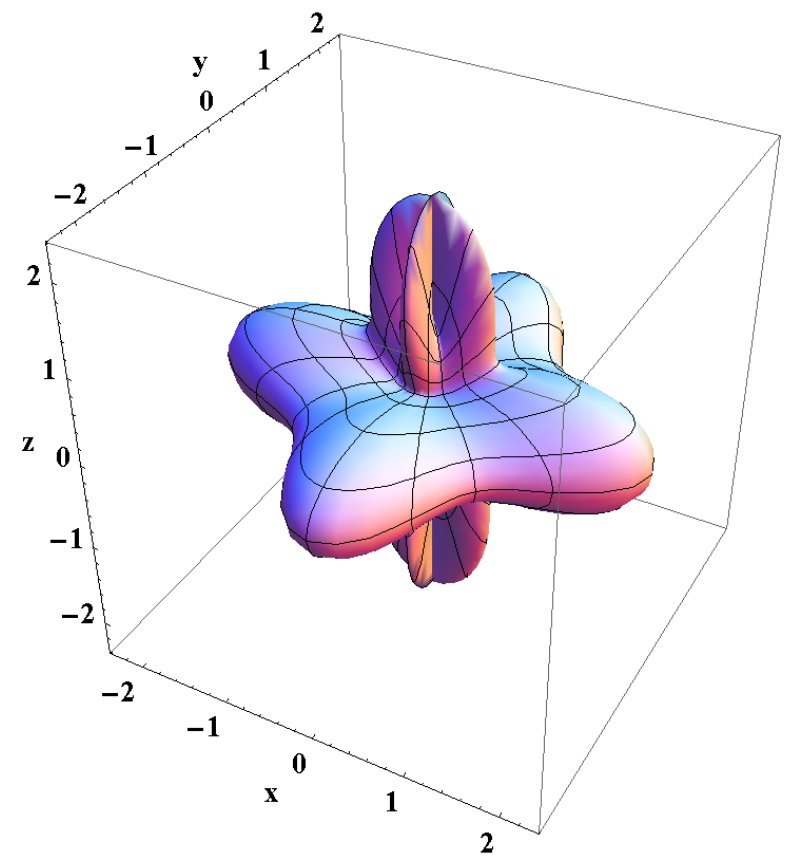

Figure $3 a$. Orientation dependence of elastic anisotropy

$$
A_{\mathrm{z}} \text { of } \mathrm{MgS}
$$

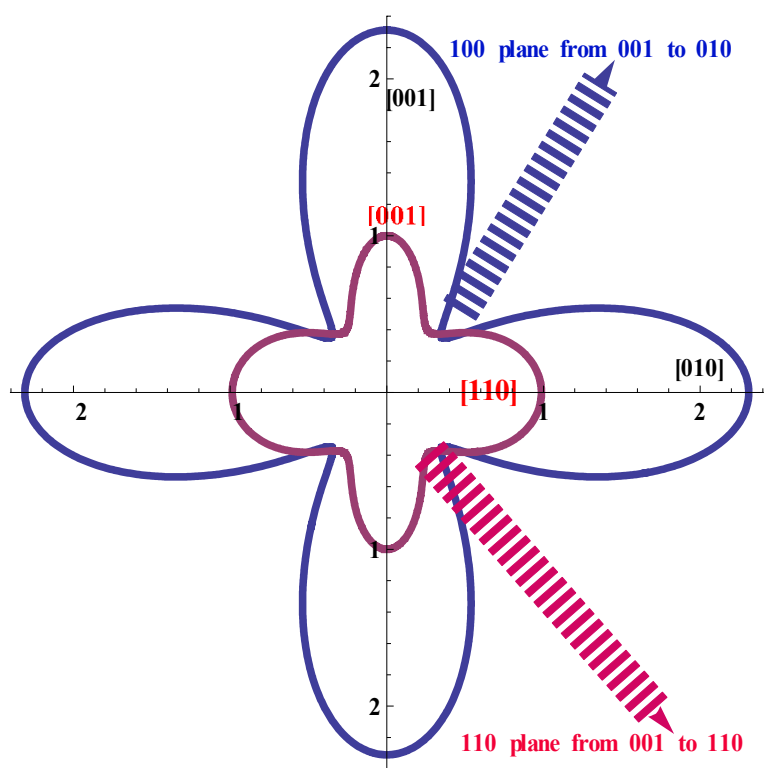

Figure $3 b$. Orientation dependence of elastic anisotropy $A_{z}$ of $\mathrm{MgS}$ in (100) and (110) planes

Figure 3. (color on line) Elastic anisotropy $A_{\mathrm{z}}$ of $\mathrm{MgS}$ with the projection maps

\subsection{The Anisotropy of Wave Propagation in $\mathrm{MgS}$}

An effective way of understanding elastic wave propagation is to evaluate the slowness surface with the three acoustic modes in that material-one longitudinal and two transverse (Hensel \& Dynes, 1979; Lax \& Narayanamurti, 1980). The slowness surface is therefore constructed by calculating the inverse phase velocity (Hammond, 1997) for all angles in $\mathbf{k}$ space. The phase velocity $\boldsymbol{v}(\mathbf{k})$ is obtained from the Christoffel equation (Koos \& Wolfe, 1984), and is derived from continuously elastic theory [36, 41]. In order to illustrate the anisotropy in elastic properties clearly, the slowness and group velocity contours have been generated using our program written in Mathematics. The inverse velocity contours or slowness surface can give more information about the elastic anisotropy. The quasi-longitudinal (QL), quasi-fast-shear (QFS) and quasi-slow-shear (QSS) modes are shown separately in these figures (Figure $4 a$ ). The contours in (100) and (1 10) planes are enough to visualize the entire surfaces, which are shown in Figure $4 b$ and $c$, respectively. The feature is QL for the inner sheet, QFS for the middle and (QSS) for the outer.

From Figure 4, it appears that the phonon focusing propagate preferentially along or near the [100] and [011] directions which are precisely the largest anisotropic direction in MgS (Figure 3). There are three obvious characteristics to appear, i.e., $(i)$ the QL-sheet contains large almost-flat regions; ( $i i)$ the QSS-sheet does contain a large region of saddle curvature than the QFS-sheet; (iii) the QSS-sheet touches the QFS-sheet at conic points (Hurley \& Wolfe, 1985; Every, 1981).

Another important physical quantity is the energy flux, $\mathbf{V}_{\mathrm{g}}=\nabla_{k} \omega(\mathbf{k})$. It associates with the given phonon and is not always collinear with its wave vector $\mathbf{k}$, and can be obtained by the phase velocity $v$ in the spherical coordinate (Every, 1981).

$$
V_{j \mathrm{x}}=n_{l}\left[v_{j}+A_{j}\left(1-2 S-n_{1}^{2}\right)+B_{j} n_{2}^{2} n_{3}^{2}\left(1-3 n_{1}^{2}\right)\right]
$$




$$
A_{j}=\frac{C_{2} C_{3}\left(2-C_{3}\right)}{\rho v_{j}} \cdot\left\{\frac{\left(b Q-\frac{a S}{2}\right) \sin \left(\psi+\frac{2 \pi}{3} j\right)}{(1-a S)^{2} \sin (3 \psi)}-\frac{\cos \left(\psi+\frac{2 \pi}{3} j\right)}{\sqrt{(1-a S)}}\right\}
$$

and

$$
B_{j}=\frac{3 C_{2} C_{3}^{2}\left(3-2 C_{3}\right)}{\rho v_{j}} \cdot \frac{\sin \left(\psi+\frac{2 \pi}{3} j\right)}{(1-a S) \sin (3 \psi)}
$$

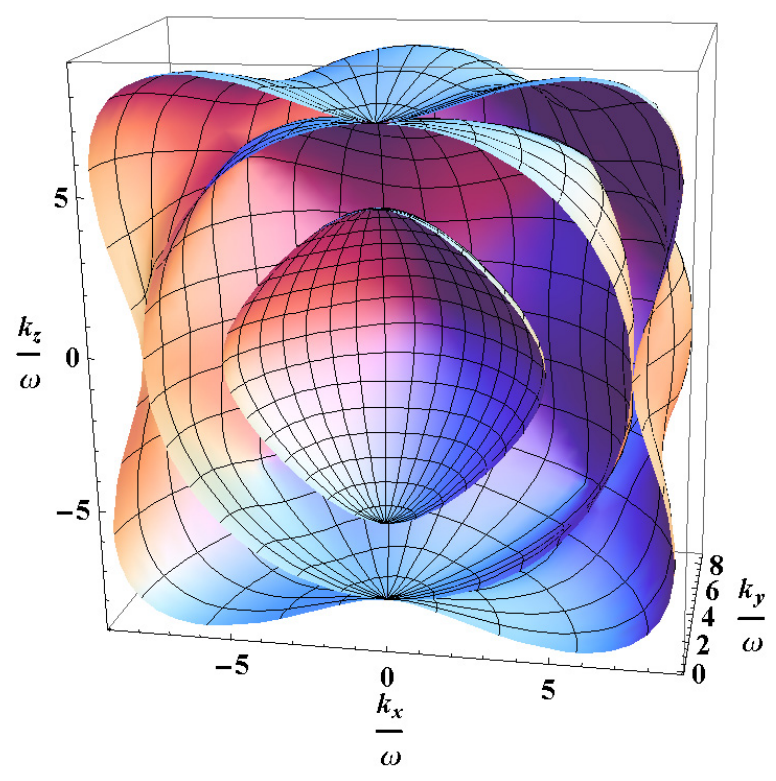

Figure $4 a$. Three-dimensional representations of the three sheets of the slowness surface in $\mathrm{MgS}$. The unit is $0.0316 \mathrm{~s} / \mathrm{km}$

$\underline{\boldsymbol{k}_{z}}$

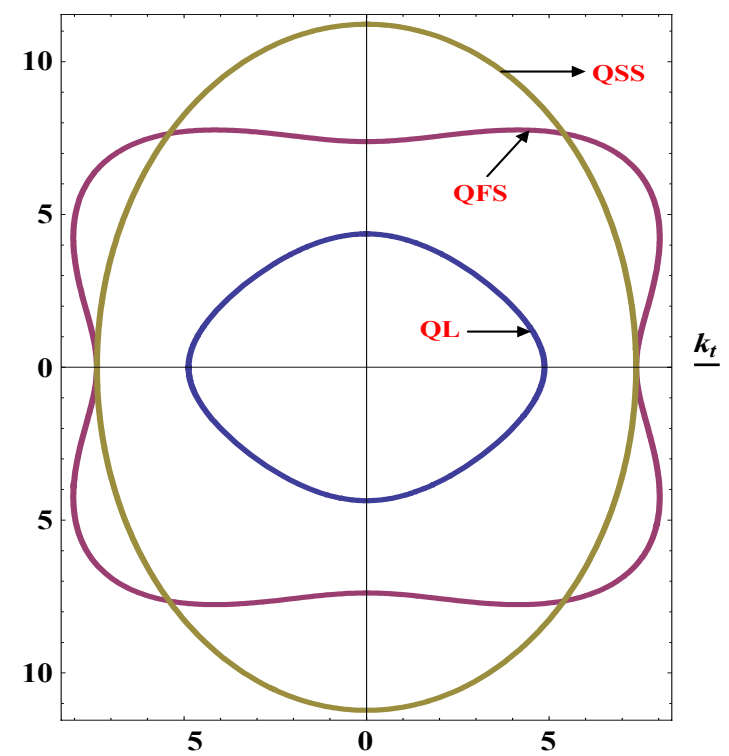

Figure 4c. T The slowness surfaces of QL, QFS and QSS of MgS on the $(\overline{1} 10)$ plane

Figure 4. (color on line)The slowness surfaces, the quasi-longitudinal (QL) for the inner sheet, quasi-fast-shear (QFS) for the middle, and quasi-slow-shear (QSS) modes for the outer. The unit is $0.0316 \mathrm{~s} / \mathrm{km}$ 
where $v_{j}$ is the phase velocity obtained from equation (7) as the polarization index $j$ takes on the values 0,1 and 2; $C_{I}=C_{11}+2 C_{44}, C_{2}=C_{11}-C_{44}$ and $\left.C_{3}=C_{11}-C_{12}-2 C_{44}\right) /\left(C_{11}-C_{44}\right) ; \psi=\frac{1}{3} \arccos \left[\frac{1-\frac{3 a \mathrm{~S}+b \mathrm{Q}}{2}}{(1-a S)^{3 / 2}}\right], S=n_{1}^{2} n_{2}^{2}+n_{1}^{2} n_{3}^{2}+n_{2}^{2} n_{3}^{2}$, $Q=n_{1}^{2} n_{2}^{2} n_{3}^{2}, a=3 C_{3}\left(2-C_{3}\right), b=\frac{27}{2} C_{3}^{2}\left(3-2 C_{3}\right) ; C_{11}, C_{12}$ and $C_{44}$ are elastic constants, $n_{1}, n_{2}$ and $n_{3}$ are the direction cosines of phase velocity.

The corresponding expressions for the y and z components of $V_{j}$ are obtained by cyclic interchange of $n_{1}, n_{2}$ and $n_{3}$.

We plotted the group velocity surfaces in order to understand more about the anisotropy in elastic properties of $\mathrm{MgS}$, which are shown in Figure 5 as sections of QL-sheet (Figure $5 a$ ), QSS-sheet (Figure $5 b$ ) and QFS-sheet (Figure $5 c$ ) together with their projections in (100) (Figure $5 d$ ) and $(\overline{1} 10)$ planes (Figure $5 e$ ). They exhibit cuspidal edges along the [100], [110] and [111] directions. It is obviously seen that the phonon propagation, particularly the quasi-shear mode is highly anisotropic in this crystal. Furthermore, the quasi-shear mode exhibits cuspidal edges along [100], [111] and [011] directions which are extreme directions of anisotropy discussed above. On the one hand, the three-dimensional group velocity surfaces and slowness surfaces have some kind of inevitable connections with the crystal anisotropy, and are affected obviously by the elastic anisotropy, on the other hand, the calculated the phonon caustics show that the phonon caustics mainly "walk along" the local extreme directions of the elastic anisotropy, which is the interesting phenomenon not founded before. However, the cuspidal edges correspond to directions which a one-to-one correspondence between phase velocity vector and group velocity vector is lost, or more than one group velocity corresponds to a single phase velocity. These features are also reflected in the slowness surfaces, and are expected to exhibit interesting phenomena such as conical refraction, phonon focusing etc. (Auld, 1973).

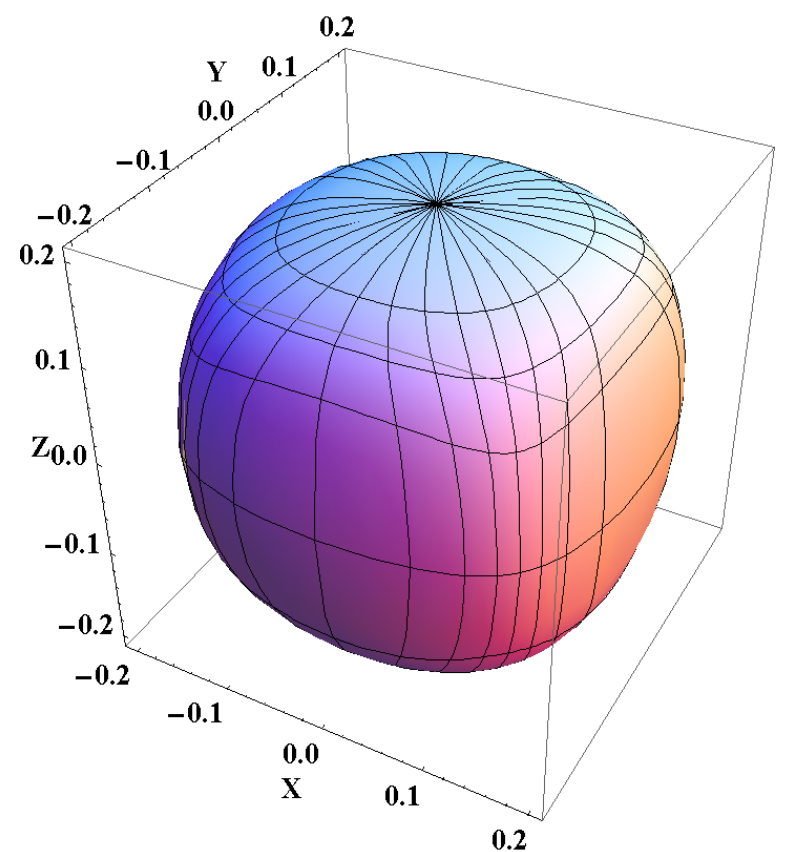

Figure $5 a$. The group velocity surface of $\mathrm{MgS}$ for QL surface

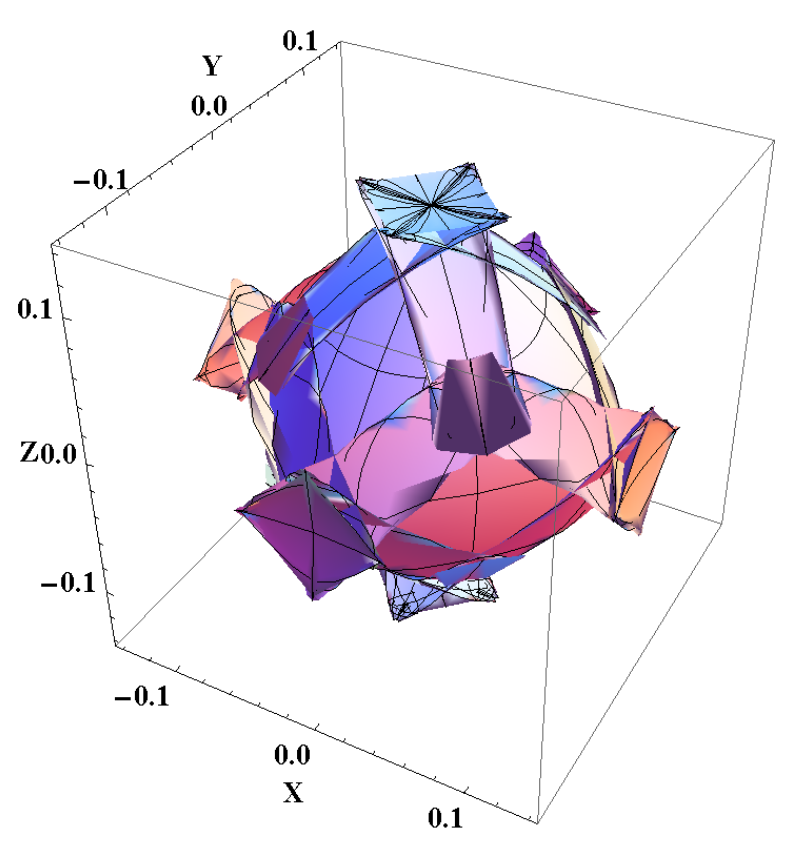

Figure $5 b$. The group velocity surface of $\mathrm{MgS}$ for the QSS surface 


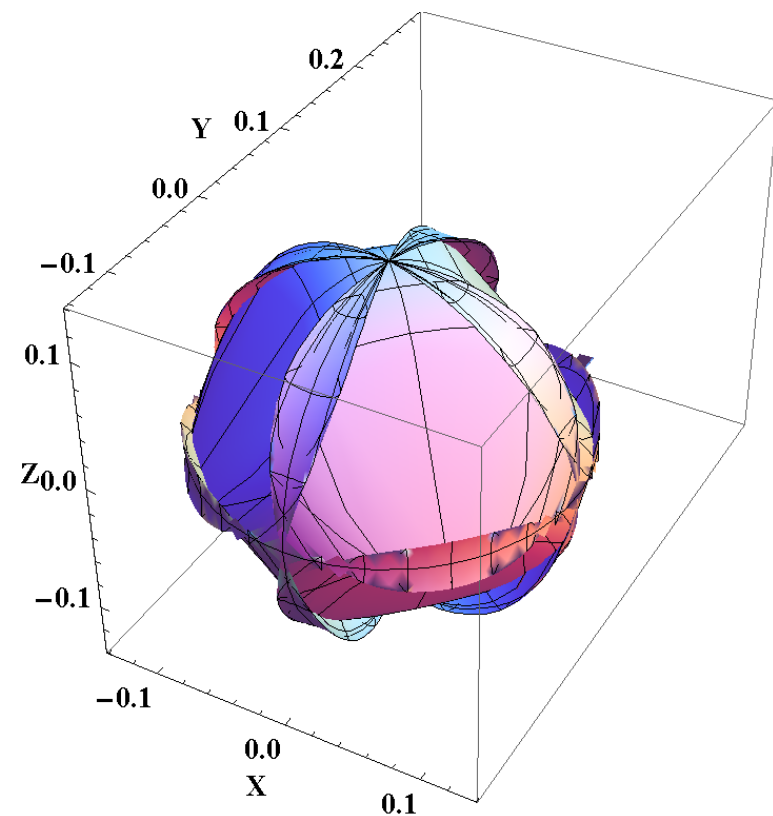

Figure $5 c$. The group velocity surface of $\mathrm{MgS}$ for the QFS surface

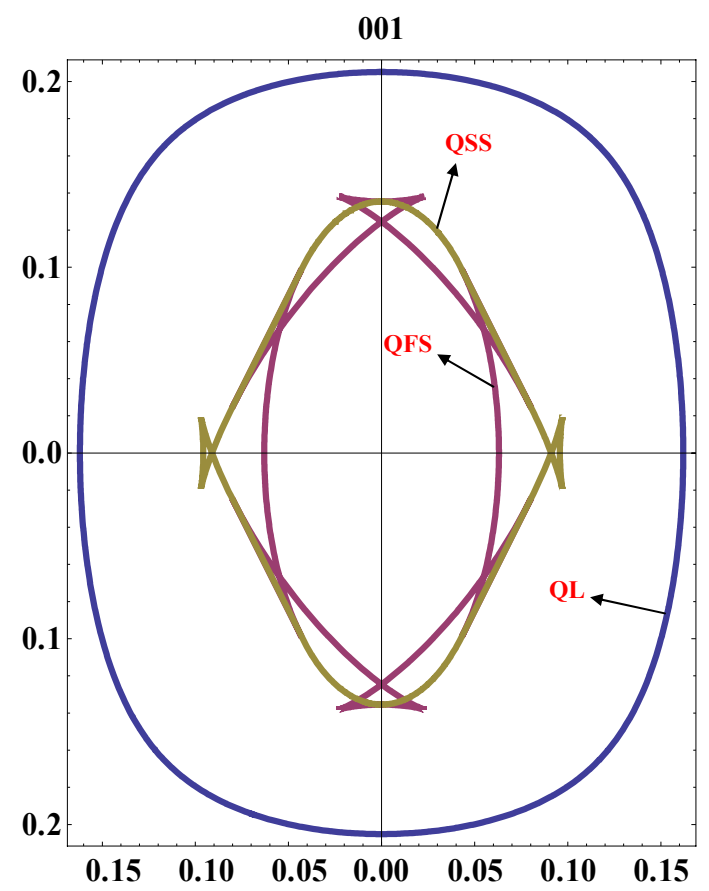

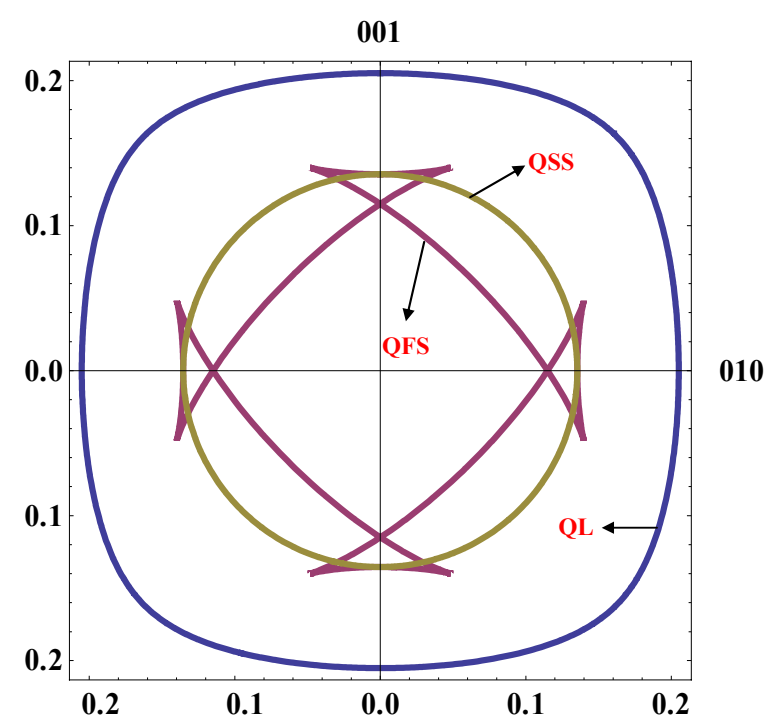

Figure $5 d$. The cross sections of group velocity surface of $\mathrm{MgS}$ on the (100) plane

Figure $5 e$. The cross sections of group velocity surface of

$$
\mathrm{MgS} \text { on the (110) plane }
$$

Figure 5. (color on line)The group velocity surface of MgS. The quasi-longitudinal (QL) for the inner sheet, quasi-fast-shear (QFS) for the middle, and quasi-slow-shear (QSS) modes for the outer. The unit is $31.622 \mathrm{~km} / \mathrm{s}$

\section{Conclusions}

Just as the bonds between atoms are anisotropic, so is the velocity of an elastic wave in the crystalline medium. We give systematically theoretical investigations of the anisotropy and phonon-focusing patterns for $\mathrm{MgS}$. To provide specific examples of the focusing features typical of each regime, the slowness surfaces of $\mathrm{MgS}$ were examined in detail. The phonon-focusing patterns are observed through the slowness surface of transverse modes. The 
quasi-fast-shear mode plays a major role for the phonon-focusing patterns followed by the quasi-slow-shear mode, and the quasi-longitudinal mode has almost no effect on the phonon-focusing patterns. The qualitative changes that take place in the focusing pattern as the elastically anisotropic properties are varied have been investigated by studying the evolution in the slowness-surface topology and by the corresponding changes in elastic anisotropy simulations. Investigations between the slowness-surface topology and the elastic anisotropy reveal distinct trends in the phonon-focusing structures. Theoretical researches for the three-dimensional slowness surface and the three-dimensional anisotropy give insights into the mixing of longitudinal and transverse mode, and show that the phonon caustics mainly "walk along" the local extremum directions of the elastic anisotropy for $\mathrm{MgS}$.

\section{Acknowledgment}

This project was supported by the Natural Science Foundation of the Education Department of Henan province of China under grant No.14B140010, and supported by National Natural Science Foundation of China (No.51471082).

\section{References}

Auld, B. A. (1973). Acoustic fields and waves in solids. New York: John Wiley \& Sons.

Bond, W. L. (1976). Crystal technology. New York: John Wiley \& Sons.

Chakrabarti, A. (2000). Role of NiAs phase in pressure-induced structural phase transitions in IIA-VI chalcogenides. Phys. Rev. B, 62, 1806. http://dx.doi.org/10.1103/PhysRevB.62.1806

Chen, Z. J., Xiao, H. Y., \& Zu, X. T. (2005). Density functional theory investigation on structural properties of MgS crystal. Acta Phys. Sin., 54, 5301. Retrieved from http:/wulixb.iphy.ac.cn/EN/abstract/abstract10230.shtml

Ching, W. Y., Gan, F. Q., \& Huang, M. Z. (1995). Band theory of linear and nonlinear susceptibilities of some binary ionic insulators. Phys. Rev. B, 52, 1596. http://dx.doi.org/10.1103/PhysRevB.52.1596

Côté, M., Zakharov, O., Rubio, A., \& Cohen, M. L. (1997). Ab initio calculations of the pressure-induced structural phase transitionsfor four II-VI compounds. Phys. Rev., B, 55, 13025. http://dx.doi.org/10.1103/ PhysRevB.55.13025

Drief, F., Tadjer, A., Mesri, D., \& Aourag, H. (2004). First principles study of structural, electronic, elastic and optical properties of $\mathrm{MgS}, \mathrm{MgSe}$ and MgTe. Catal. Today, 89, 343. Retrieved from http://www.sciencedirect.com/science/article/pii/S0920586103006515

Duman, S., Bagc, S., Tutuncu, H. M., \& Srivastava, G. P. (2006). (First-principles studies of ground-state and dynamical properties of $\mathrm{MgS}, \mathrm{MgSe}$, and $\mathrm{MgTe}$ in the rocksalt, zinc blende, wurtzite, and nickel arsenide phases). Phys. Rev. B, 73, 205201. http://dx.doi.org/10.1103/PhysRevB.73.205201

Every, A. G. (1979). General, Closed-Form Expressions for Acoustic Waves in Cubic Crystals. Phys. Rev. Lett., 42, 1065. http://dx.doi.org/10.1103/PhysRevLett.42.1065

Every, A. G. (1980). General closed-form expressions for acoustic waves in elastically anisotropic solids. Phys. Rev. B, 22, 1746. http://dx.doi.org/10.1103/PhysRevB.22.1746

Every, A. G. (1981). Ballistic phonons and the shape of the ray surface in cubic crystals. Phys. Rev. B, $24,3456$. http://journals.aps.org/prb/abstract/10.1103/PhysRevB.24.3456

Every, A. G., Sachse, W., Kim, K. Y., \& Thompson, M. O. (1990). Epitaxy-induced structural phase transformations. Phys. Rev. Lett., 65, 1446. http://journals.aps.org/prb/abstract/10.1103/PhysRevB.38.10124

Froyen, S., Wei, S. H., \& Zunger, A. (1988). Epitaxy-induced structural phase transformations. Phys. Rev. B, 38, 10124. http://dx.doi.org/10.1103/PhysRevB.38.10124

Hammond, C. (1997). The Basics of Crystallography \& Diffraction. Oxford: Oxford University Press.

Hauser, M. R., Weaver, R. L., \& Wolfe, J. P. (1992). Internal diffraction of ultrasound in crystals: Phonon focusing at long wavelengths. Phys. Rev. Lett., 68, 2604. http://dx.doi.org/10.1103/PhysRevLett.68.2604

Hensel, J. C., \& Dynes, R. C. (1977). Interaction of Electron-Hole Drops with Ballistic Phonons in Heat Pulses: The Phonon Wind. Phys. Rev. Lett., 39, 969. http://dx.doi.org/10.1103/PhysRevLett.39.969

Hensel, J. C., \& Dynes, R. C.(1979). Observation of Singular Behavior in the Focusing of Ballistic Phonons in Ge. Phys. Rev. Lett., 43, 1033. http://dx.doi.org/10.1103/PhysRevLett.43.1033 
Hohenberg, P., \& Kohn, W. (1964). Inhomogeneous Electron Gas. Phys. Rev , 136, B864. http://journals.aps.org/ pr/abstract/10.1103/PhysRev.136.B864

http://www.sciencedirect.com/science/article/pii/0038109894903395

Hurley, D. C., \& Wolfe, J. P. (1985). Phonon focusing in cubic crystals. Phys. Rev. B, 32, 2568. http://dx.doi.org/10.1103/PhysRevB.32.2568

Hurley, D. C., \& Wolfe, J. P. (1985). Phonon focusing in cubic crystals. Phys. Rev. B, 32, 2568. http://dx.doi.org/10.1103/PhysRevB.32.2568

Hurley, D. C., Wolfe, J. P., \& McCarthy, K. A. (1986). Phonon focusing in tellurium dioxide. Phys. Rev. B, 33, 4189. http://dx.doi.org/10.1103/PhysRevB.33.4189

Kalpana, G., Palanivel, B., Thomas, R. M., \& Rajagopalan, M. (1996). Electronic and structural properties of MgS and MgSe. Physica B, 222, 223. http://www.sciencedirect.com/science/article/pii/0921452696000142

Kohn, W., \& Sham, L. J. (1965). Quantum Density Oscillations in an Inhomogeneous Electron Gas. Phys. Rev., 137, A1697. http://journals.aps.org/pr/abstract/10.1103/PhysRev.137.A1697

Koos, G. L., \& Wolfe, J. P. (1984). Piezoelectricity and ballistic heat flow. Phys. Rev. B, $29,6015$. http://dx.doi.org/10.1103/PhysRevB.29.6015

Lax, M., \& Narayanamurti, V. (1980). General closed-form expressions for acoustic waves in elastically anisotropic solids. Phys. Rev. B., 22, 1746. http://dx.doi.org/10.1103/PhysRevB.22.1746

Lee, S. G., \& Chang, K. J. (1995). First-principles study of the structural properties of MgS-, MgSe-, ZnS-, and ZnSe-based superlattice. Phys. Rev. B, 52, 1918. http://dx.doi.org/10.1103/PhysRevB.52.1918

Lichanot, A., Dargelos, A., Larrie, C., \& Orlando, R. (1994). Electronic structure and phase transition in magnesium sulphide. Solid StateCommun, 90, 189.

Maris, H. J. (1971). Enhancement of Heat Pulses in Crystals due to Elastic Anisotropy. J. Acoust. Soc. Am., 50, 812. http://scitation.aip.org/content/asa/journal/jasa/50/3B/10.1121/1.1912705

Maris, H. J. (1983). Effect of finite phonon wavelength on phonon focusing. Phys. Rev. B, $28,7033$. http://dx.doi.org/10.1103/PhysRevB.28.7033

Monkhorst, H. J. \& Pack, J. D. (1976). Special points for Brillouin-zone integrations. Phys. Rev. B, 13, 5188. http://dx.doi.org/10.1103/PhysRevB.13.5188

Northrop, G. A., \& Wolfe, J. P. (1980). Ballistic phonon imaging in germanium. Phys. Rev. B, 22, 6196. http://dx.doi.org/10.1103/PhysRevB.22.6196

Perdew, J. P., Burke, K., \& Ernzerhof, M. (1996). Generalized Gradient Approximation Made Simple. Phys. Rev. Lett, 77, 3865. http://dx.doi.org/10.1103/PhysRevLett.77.3865

Rached, D., Benkhettou, N., Soudini, B., Abbar, B., Sekkal, N., \& Driz, M. (2003). Electronic structure calculationsof magnesium chalcogenides $\mathrm{MgS}$ and MgSe. Phys. stat. sol. (b), 240, 565. http://onlinelibrary. wiley.com/doi/10.1002/pssb.200301889/abstract

Sahraoui, F. A., Zerroug, S., Louail, L., \& Maouche, D. (2007). Effect of pressure on the structural and elastic properties of $\mathrm{ZnS}$ and $\mathrm{MgS}$ alloys in the B3 and B1 phases. Mater. Lett., 61, 1978. Retrieved from http://www.sciencedirect.com/science/article/pii/S0167577X06010007

Taylor, B., Maris, H. J., \& Elbaum, C. (1969). Phonon Focusing in Solids. Phys. Rev. Lett., 23, 416. http://dx.doi.org/10.1103/PhysRevLett.23.416

Vanderbilt, D. (1990). Soft self-consistent pseudopotentials in a generalized eigenvalue formalism. Phys. Rev. B, 41, 7892. http://dx.doi.org/10.1103/PhysRevB.41.7892

Varshney, D., Kaurav, N., Sharma, U., \& Singh, R. K. (2008). Phase transformation and elastic behavior of MgX ( $\mathrm{X}=\mathrm{S}, \mathrm{Se}, \mathrm{Te})$ alkaline earth chalcogenides. J. Phys. Chem. Solids, 69, 60. Retrieved from http://www.sciencedirect.com/science/article/pii/S0022369707004490

Weilert, M. A., Msall, M. E., Anderson, A. C., \& Wolfe, J. P. (1993). Phonon scattering from ferroelectric domain walls: Phonon imaging in KDP. Phys. Rev. Lett., 71, 735. http://journals.aps.org/prl/abstract/10.1103/ PhysRevLett.71.735

Wolfe, J. P. (1998). Imaging Phonons (pp. 106-116). Cambridge, UK: Cambridge University Press. 
Wolverson, D., Bird, D. M., Bradford, C., Prior, K. A., \& Cavenett, B. C. (2001). Lattice dynamics and elastic properties of zinc-blende MgS. Phys. Rev. B, 64, 113203. http://dx.doi.org/10.1103/PhysRevB.64.113203

Würz, K.U, Wesner, J., Hillmann, K., \& Grill, W. (1995). Determination of elastic constants using a scanning acoustic microscope. Z. Phys. B, 97, 487. http://link.springer.com/article/10.1007/BF01322428

Zener, C. (1948). Elasticity \& Anelasticity of Metals. Chicago: University Chicago Press.

Zimmer, H. G., Winzen, H., \& Syassen (1985). High-pressure phase transitions in CaTe and SrTe. Phys. Rev. B, 32, 4066. http://dx.doi.org/10.1103/PhysRevB.32.4066

\section{Copyrights}

Copyright for this article is retained by the author(s), with first publication rights granted to the journal.

This is an open-access article distributed under the terms and conditions of the Creative Commons Attribution license (http://creativecommons.org/licenses/by/3.0/). 